\title{
ON DIRECT PRODUCT MATRICES $\dagger$
}

\author{
BY W. E. ROTH
}

1. Introduction. If $A=\left(a_{i j}\right),(i=1,2, \cdots, m ; j=1,2, \cdots, n)$, is an $m \times n$ matrix and $B$ is a $p \times q$ matrix, then the matrix, $P=\left(a_{i j} B\right)=A\langle B\rangle$, of order $m p \times n q$, whose elements occur in $m n$ blocks, $a_{i j} B$, is the direct product of $A$ and $B . \ddagger$

In the present paper we determine the elementary divisors of $A\langle B\rangle-\lambda I$ and of $\rho A\langle I\rangle+\sigma I\langle B\rangle-\lambda I$, where $\rho$ and $\sigma$ are scalar constants, and where the elementary divisors of $A-\lambda I$ and of $B-\lambda I$ are known. Finally, $\$ 3$ takes up the discussion of the linear matrix equation

$$
A_{1} X_{1} B_{1}+A_{2} X_{2} B_{2}+\cdots+A_{r} X_{r} B_{r}=C .
$$

The reduction of this equation to an equation whose solution is known is accomplished by means of direct product matrices and thus perfects a procedure first noted by MacDuffee. $\$$

2. On Elementary Divisors of $A\langle B\rangle-\lambda I$ and of $\rho A\langle I\rangle+$ $\sigma I\langle B\rangle-\lambda I$. In this section it will be convenient to indicate the order of a matrix by subscripts; thus $A_{\alpha, \beta}$ is an $\alpha \times \beta$ matrix, $B_{p}$ is a square matrix of order $p$, and $I_{p}$ is the unit matrix of order $p$. Matrices will be designated throughout by capitals, whereas lower case letters will be employed to denote scalars, such as parameters, constants, and the elements of matrices. Moreover, all scalars will be regarded as belonging to the complex number field. Hence, for our purpose, $\left(a_{i j} B\right)=\left(B a_{i j}\right)=A\langle B\rangle$ $=\langle B\rangle A$.

$\dagger$ Presented to the Society, April 6, 1934.

$\ddagger$ Zehfuss, Ueber eine gewisse Determinante, Zeitschrift für Mathematik und Physik, 3te Jahrgang (1858), pp. 298-301, was perhaps the first to study determinants of this form. Rutherford, On the condition that two Zehfuss matrices be equal, this Bulletin, vol. 39 (1933), pp. 801-808, called $P$ the Zehfuss matrix of $A$ and $B$ and devised the notation here employed. Dickson, Algebras and their Arithmetics, 1923, p. 119, and MacDuffee, The Theory of Matrices, 1933, p. 81, employ the term direct product to designate $P$. The reader is also referred to the latter treatise for a more complete discussion of direct product matrices.

$\S$ MacDuffee, loc. cit., p. 89. 
If $A_{\alpha, \beta}=\left(a_{i j}\right), C_{\beta, \gamma}=\left(c_{i j}\right), B_{a, b}$, and $D_{b, c}$ are given matrices, then

$$
\begin{aligned}
& A_{\alpha, \beta}\left\langle B_{a, b}\right\rangle C_{\beta, \gamma}\left\langle D_{b, c}\right\rangle=\left(a_{i j} B_{a, b}\right)\left(c_{j k} D_{b, c}\right) \\
= & \left(\sum_{j=1}^{\beta} a_{i j} c_{j k} B_{a, b} D_{b, c}\right)=A_{\alpha, \beta} C_{\beta, \gamma}\left\langle B_{a, b} D_{b, c}\right\rangle .
\end{aligned}
$$

This identity was first proved by Stéphanos. $\dagger$ Evidently, if $Q_{m}$ and $R_{n}$ are non-singular matrices, then $Q_{m}\left\langle I_{n}\right\rangle$ and $I_{m}\left\langle R_{n}\right\rangle$ are likewise non-singular matrices. Hence, according to (1),

$$
Q_{m}\left\langle I_{n}\right\rangle \cdot I_{m}\left\langle R_{n}\right\rangle=I_{m}\left\langle R_{n}\right\rangle \cdot Q_{m}\left\langle I_{n}\right\rangle=Q_{m}\left\langle R_{n}\right\rangle ;
$$

and $Q_{m}\left\langle R_{n}\right\rangle$, being the product of non-singular matrices, is also non-singular. If $Q_{m}^{I}$ is the inverse of $Q_{m}$ and $R_{n}^{I}$ that of $R_{n}, \ddagger$ then $Q_{m}^{I}\left\langle R_{n}^{I}\right\rangle$ is the inverse of $Q_{m}\left\langle R_{n}\right\rangle, \S$ for by (1),

$$
Q_{m}\left\langle R_{n}\right\rangle \cdot Q_{m}{ }^{I}\left\langle R_{n}{ }^{I}\right\rangle=I_{m}\left\langle I_{n}\right\rangle=I_{m n} .
$$

Let $Q_{m}$ and $R_{n}$ be the non-singular matrices which transform $A_{m}$ and $B_{n}$ to their respective normal forms, $A_{m}{ }^{*}$ and $B_{n}{ }^{*}$. That is,

$$
Q_{m} A_{m} Q_{m}{ }^{I}=A_{m}^{*}, \quad R_{n} B_{n} R_{n}{ }^{I}=B_{n}^{*}
$$

where

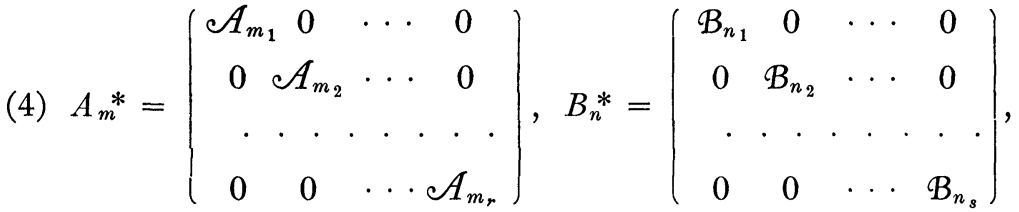

and

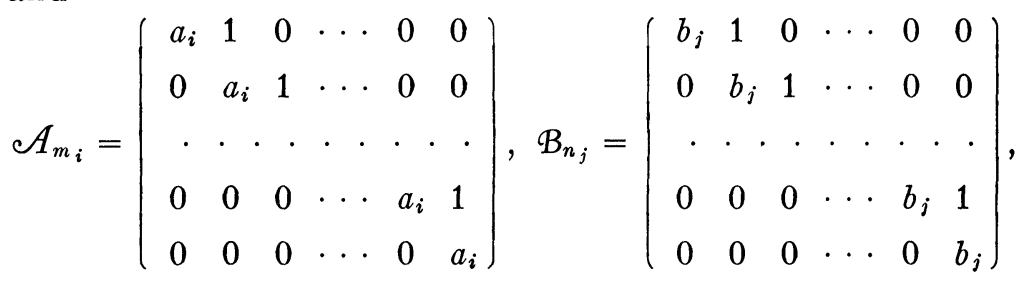

† Stéphanos, Sur une extension du calcul des substitutions linéaires, Journal de Mathématiques, (5), vol. 6 (1900), pp. 73-128.

$\ddagger$ This notation for inverse is due to MacDuffee, loc. cit.

$\S$ Stéphanos, loc. cit. 
$(i=1,2, \cdots, r ; j=1,2, \cdots, s)$. Consequently $A_{m}-\lambda I_{m}$ has the elementary divisors $\left(a_{i}-\lambda\right)^{m_{i}},(i=1,2, \cdots, r)$, and $B_{n}-\lambda I_{n}$ has the elementary divisors $\left(b_{j}-\lambda\right)^{n_{j}},(j=1,2, \cdots, s)$. We shall prove the following theorem.

THEOREm 1. If $A_{m}-\lambda I_{m}$ and $B_{n}-\lambda I_{n}$ have the elementary divisors $\left(a_{i}-\lambda\right)^{m_{i}},(i=1,2, \cdots, r)$, and $\left(b_{j}-\lambda\right)^{n_{j}},(j=1,2, \cdots, s)$, respectively, and if $\mu_{i j}$ is the lesser of the integers $m_{i}$ and $n_{j}$ or their common value, then the matrix $A_{m}\left\langle B_{n}\right\rangle-\lambda I_{m n}$ has the elementary divisors

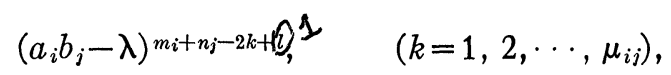

if $a_{i} b_{j} \neq 0, \lambda^{m_{j}}$ occurring $n_{j}$ times, if $a_{i}=0, b_{j} \neq 0, \lambda^{n_{j}}$ occurring $m_{i}$ times, if $a_{i} \neq 0, b_{j}=0$, and $\lambda, \lambda^{2}, \cdots, \lambda^{\mu_{i j-1}}$ each occurring twice and $\lambda^{\mu_{i j}}$ occurring $m_{i}+n_{j}-2 \mu_{i j}+1$ times if $a_{i}=b_{j}=0, \quad(i=1$, $2, \cdots, r ; j=1,2, \cdots, s)$.

Now by (1), (2), and (3), we have

$$
Q_{m}\left\langle R_{n}\right\rangle\left[A_{m}\left\langle B_{n}\right\rangle-\lambda I_{m n}\right] Q_{m}^{I}\left\langle R_{n}{ }^{\prime}\right\rangle=A_{m}{ }^{*}\left\langle B_{n}^{*}\right\rangle-\lambda I_{m n} ;
$$

consequently the elementary divisors of $A_{m}\left\langle B_{n}\right\rangle-\lambda I_{m n}$ are identical with those of $A_{m}{ }^{*}\left\langle B_{n}{ }^{*}\right\rangle-\lambda I_{m n}$. Upon writing out the latter matrix, we readily see that its elementary divisors are identical with the aggregate of those of the $r$ matrices

$$
A_{m_{i}}\left\langle B_{n}^{*}\right\rangle-\lambda I_{m_{i} n}, \quad(i=1,2, \cdots, r),
$$

each taken separately. By an appropriate interchange of rows and of columns the matrix $A_{m_{i}}\left\langle B_{n}{ }^{*}\right\rangle-\lambda I_{m_{i n}}$ can be transformed to an equivalent matrix having the $m_{i} n_{j} \times m_{i} n_{j}$ matrices $A_{m_{i}}\left\langle B_{n_{i}}\right\rangle-\lambda I_{m_{i} n_{j}},(j=1,2, \cdots, s)$, in its principal diagonal and zero matrices elsewhere. Hence the elementary divisors of $A_{m}\left\langle B_{n}\right\rangle-\lambda I_{m n}$ are identical with the aggregate of those of the $r s$ matrices

$$
A_{m_{i}}\left\langle\mathcal{B}_{n_{j}}\right\rangle-\lambda I_{m_{i} n_{j}}, \quad(i=1,2, \cdots r ; j=12, \cdots, s),
$$

each taken independently.

If $b_{j} \neq 0, \mathcal{B}_{n_{j}}$ is a non-singular matrix of order $n_{j}$; consequently, by the method of Bôcher, $\dagger$

$$
A_{m_{i}}\left\langle B_{n_{j}}\right\rangle-\lambda I_{m_{i} n_{j}}
$$

† Bôcher, Introduction to Higher Algebra, 1922, §\$91 and 92. 
has $\left(m_{i}-1\right) n_{j}$ unit invariant factors and its elementary divisors in $\lambda$ are identical with those of $\left(a_{i} B_{n_{j}}-\lambda I_{n_{j}}\right)^{m_{i}}$. If we let $\mu_{i j}$ be the lesser of the two integers $m_{i}$ and $n_{j}$ or their common value, this matrix has the elementary divisors $\left(a_{i} b_{j}-\lambda\right)^{m_{i}+n_{j-2 k+1}}$, $\left(k=1,2, \cdots, \mu_{i j}\right)$, if $a_{i} \neq 0$, and the elementary divisor $\lambda^{m_{i}}$ occurring $n_{j}$ times, if $a_{i}=0$. This establishes the first two cases under the above theorem.

If $b_{j}=0$ and $a_{i} \neq 0, A_{m_{i}}\left\langle B_{n_{j}}\right\rangle$ is a nilpotent matrix such that its $n_{j}$ th power is zero but no lower power vanishes. Hence, in this case, (5) has only elementary divisors of the form $\lambda^{h}$, where $h$ cannot exceed $n_{j}$. But since $a_{i} \neq 0$, (5) has a non-singular minor of order $\left(n_{j}-1\right) m_{i}$ which is independent of $\lambda$; hence at most $m_{i}$ elementary divisors can be powers of $\lambda$. The determinant of (5) is $\lambda^{m_{i} n_{j}}$, consequently the $m_{i}$ elementary divisors must be $\lambda^{n_{j}}$. This establishes the third case under the theorem.

If $a_{i}=b_{j}=0, A_{m_{i}}\left\langle\mathcal{B}_{n_{j}}\right\rangle$ has $\left(m_{i}-1\right)\left(n_{i}-1\right)$ unit elements in the $\left(n_{j}+1\right)$ th diagonal above its principal diagonal and all remaining elements are zeros. Hence (5) has a non-singular minor of order $\left(m_{i}-1\right)\left(n_{j}-1\right)$ which is independent of $\lambda$, and no such minor of higher order. Consequently this matrix has $m_{\imath} n_{j}-$ $\left(m_{i}-1\right)\left(n_{j}-1\right)=m_{i}+n_{j}-1$ elementary divisors which are powers of $\lambda$. Now $A_{m_{i}}\left\langle\mathcal{B}_{n_{j}}\right\rangle$ is nilpotent of degree $\mu_{i j}$, for in the present case the $\mu_{i j}$ th power of either $A_{m_{i}}$ or of $\mathcal{B}_{n_{j}}$ vanishes but neither vanishes for powers less than $\mu_{i j}$. Consequently (5) has $m_{i}+n_{j}-1$ elementary divisors $\lambda^{k}$, whose degree $k$ does not exceed $\mu_{i j}$ and must have at least one of degree $\mu_{i j}$. We may, therefore, assume that (5) has the elementary divisors $\lambda^{k}$ occurring $\nu_{k}$ times, $\left(k=1,2, \cdots, \mu_{i_{j}}\right)$. Hence its determinant is $-\lambda$ raised to the power,

$$
\nu_{1}+2 \nu_{2}+\cdots+\mu_{i j} \nu_{\mu_{i j}}=m_{i} m_{j} .
$$

Now let the normal form of $A_{m_{i}}\left\langle\mathcal{B}_{n_{j}}\right\rangle$ be $P$, where $P$ has the form corresponding to that for $A_{m}$ and $B_{n}$ as given by (4). Then $P^{k}$ has

$$
\nu_{k+1}+2 \nu_{k+2}+\cdots+\left(\mu_{i j}-k\right) \nu_{\mu_{i j}}
$$

unities in the $k$ th diagonal above its principal diagonal and zeros elsewhere, and $\left[\mathcal{A}_{m_{i}}\left\langle\mathcal{B}_{n_{j}}\right\rangle\right]^{k}$ has $\left(m_{i}-k\right)\left(n_{j}-k\right)$ unities in the $k\left(n_{j}+1\right)$ th diagonal above its principal diagonal and 
zeros elsewhere. Since these two matrices are of the same rank for all values of $k$, we have

(7) $\nu_{k+1}+2 \nu_{k+2}+\cdots+\left(\mu_{i j}-k\right) \nu_{\mu_{i j}}=\left(m_{i}-k\right)\left(n_{j}-k\right)$,

$\left(k=1,2, \cdots, \mu_{i j}-1\right)$. The $\mu_{i j}$ equations (6) and (7) have the solution $\nu_{k}=2,\left(k=1,2, \cdots, \mu_{i j}-1\right)$, and $\nu_{\mu_{i j}}=m_{i}+n_{j}-2 \mu_{i j}$ +1 . Thus, in case $a_{i}=b_{j}=0$, (5) has the $m_{i}+n_{j}-1$ elementary divisors, stated in the above theorem.

CoRollary. Under the hypotheses of the theorem above $A_{m}\left\langle I_{p}\right\rangle-\lambda I_{m p}$ has the elementary divisors $\left(a_{i}-\lambda\right)^{m_{i}},(i=1,2$, $\cdots, r)$, each occurring $p$ times, and $I_{p}\left\langle B_{n}\right\rangle-\lambda I_{n p}$ has the elementary divisors $\left(b_{j}-\lambda\right)^{n_{j}},(j=1,2, \cdots, s)$, each occurring $p$ times.

This corollary follows directly from Theorem 1, for in this case, the matrix $I_{p}-\lambda I_{p}$ has the elementary divisor $1-\lambda$ occurring $p$ times, hence $\mu_{i j}=1,(j=1,2, \cdots, p)$, in the present case. Of course, this corollary is obviously true without recourse to the above theorem.

THEOREM 2. Under the hypotheses of Theorem 1, the matrix

$$
\rho A_{m}\left\langle I_{n}\right\rangle+\sigma I_{n}\left\langle B_{n}\right\rangle-\lambda I_{m n},
$$

where $\rho$ and $\sigma$ are scalar constants not zero, has the elementary divisors $\left(\rho a_{i}+\sigma b_{j}-\lambda\right)^{m_{i}+n_{j}-2 k+1},\left(k=1,2, \cdots, \mu_{i j} ; i=1,2, \cdots, r\right.$; $j=1,2, \cdots, s)$.

By an argument parallel to that followed in the proof of Theorem 1 we can show that the elementary divisors of $\rho A_{m}\left\langle I_{n}\right\rangle$ $+\sigma I_{m}\left\langle B_{n}\right\rangle-\lambda I_{m n}$ are identical with the aggregate of those of the $r s$ matrices

$\left[\rho a_{i} I_{n_{j}}+\sigma \mathcal{B}_{n_{j}}-\lambda I_{n_{j}}\right]^{m_{i}}, \quad(i=1,2, \cdots, r ; j=1,2, \cdots, s)$.

Hence the elementary divisors of (8) are those given by Theorem 2, if neither $\rho$ nor $\sigma$ is zero. The case where $\rho$ or $\sigma$ is zero is covered by the Corollary above. $\dagger$

$\dagger$ Recently the author learned that A. C. Aitken, in a paper that will appear soon in the Proceedings of the London Mathematical Society, gives results quite closely analogous to those of Theorem 1. 
3. The Linear Matrix Equation. We shall take up briefly the linear matrix equation

$$
\sum_{i=1}^{r} A_{i} X_{i} B_{i}=C
$$

where $A_{i}$ are $m \times n_{i}$ matrices; $B_{i},(i=1,2, \cdots, r)$, are $n_{i} \times n$ matrices; and $C$ is an $m \times n$ matrix, all known; while $X_{i},(i=1$, $2, \cdots, r)$, are unknown $m_{i} \times m_{i}$ matrices. $\dagger$ We here transform this equation to an equivalent equation by means of the matrix $M^{R}$ and direct product matrices, where $M^{R}$ is defined as follows: Let $M=\left(m_{i j}\right)$ be an $\alpha \times \beta$ matrix and let

$$
M_{i}=\left(m_{i 1}, m_{i 2}, \cdots, m_{i \beta}\right), \quad(i=1,2, \cdots, \alpha) ;
$$

then $M^{R}=\left(M_{1}, M_{2}, \cdots, M_{\alpha}\right)$, and is consequently a one-rowed matrix of $\alpha \beta$ elements. Similarly $M^{C}$ is the matrix resulting if the second, third, $\cdots$, last column of $M$ be written each below the next preceding column; thus it is an $\alpha \beta \times 1$ matrix. We can then show that $M^{C R}=M^{T R}, M^{R T}=M^{T C}$, where $M^{C T}$ is the transpose of $M^{C}$, etc. Let

$$
Y_{i}=A_{i} X_{i}, \quad Z_{i}=Y_{i} B_{i}, \quad(i=1,2, \cdots, r)
$$

then $Z_{i}=A_{i} X_{i} B_{i}$. We can readily show from (10) that

$$
Y_{i}^{R}=X_{i}^{R}\left[A_{i}^{T}\left\langle I_{n_{i}}\right\rangle\right], \quad Z_{i}^{R}=Y_{i}^{R}\left[I_{m}\left\langle B_{i}\right\rangle\right],
$$

where $A_{i}{ }^{T}$ is the transpose of $A_{i}$. Hence, by (1),

$$
Z_{i}^{R}=X_{i}^{R}\left[A_{i}^{T}\left\langle I_{n i}\right\rangle\right] \cdot\left[I_{m}\left\langle B_{i}\right\rangle\right]=X_{i}^{R}\left[A_{i}^{T}\left\langle B_{i}\right\rangle\right],
$$

where $A_{i}{ }^{T}\left\langle B_{i}\right\rangle$ is then an $m_{i} n_{i} \times m n$ matrix. With this transformation, equation (9) becomes $\sum_{i=1}^{r} X_{i}^{R} A_{i}{ }^{T}\left\langle B_{i}\right\rangle=C^{R}$, or

$$
\left(X_{1}{ }^{R}, X_{2}{ }^{R}, \cdots, X_{r}{ }^{R}\right)\left(\begin{array}{c}
A_{1}{ }^{T}\left\langle B_{1}\right\rangle \\
A_{2}{ }^{T}\left\langle B_{2}\right\rangle \\
\vdots \\
A_{r}^{T}\left\langle B_{r}\right\rangle
\end{array}\right)=C^{R} .
$$

That is, (9) is thereby transformed to an equivalent equation whose theory is well known, for it is a system of $m n$ linear equa-

$\dagger$ MacDuffee, loc. cit., discusses this equation for the case where the $A_{i}$ and $B_{i}$ are $n \times n$ matrices and gives adequate references. He extends the theory in the direction we take here, but introduces an error which will be explained below. 
tions in $\sum_{i=1}^{r} m_{i} n_{i}$ unknowns and has a solution provided the matrix of the coefficients and of this matrix augmented by $C^{R}$ have the same rank. $\dagger$ Hence we may state the following result.

THEOREM 3. A necessary and sufficient condition that the equation (9) where $A_{i}$ are $m \times m_{i}, B_{i}$ are $n_{i} \times n$ matrices, and $C$ is an $m \times n$ matrix, all known, while $X_{i},(i=1,2, \cdots, r)$ are unknown $m_{i} \times n_{i}$ matrices, have a solution, is that the matrices

$$
P=\left(\begin{array}{c}
A_{1}^{T}\left\langle B_{1}\right\rangle \\
A_{2}^{T}\left\langle B_{2}\right\rangle \\
\vdots \\
A_{r}^{T}\left\langle B_{r}\right\rangle
\end{array}\right) \text { and }\left(\begin{array}{c}
C^{R} \\
P
\end{array}\right), \quad C \neq 0
$$

have the same rank, and in case $C=0$, a necessary and sufficient condition that (9) have a solution, $X_{i},(i=1,2, \cdots, r)$, not all zero is that the rank of $P$ be less than $\sum_{i-1}^{r} m_{i} n_{i}$.

In case $X_{1}=X_{i},(i=2,3, \cdots, r),(11)$ becomes

$$
X_{1}{ }^{R}\left[\sum_{i=1}^{r} A_{i}^{T}\left\langle B_{i}\right\rangle\right]=C^{R}
$$

which is a system of $m n$ linear equations in the $m_{1} n_{1}$ unknown elements of $X_{1}$. This leads to the following theorem.

THEOREM 4. A necessary and sufficient condition that the equation

$$
\sum_{i=1}^{r} A_{i} X B_{i}=C
$$

where $A_{i}$ are $m \times n$ matrices, $B_{i}$ are $p \times q$ matrices, and $C$ is an $m \times q$ matrix, and where $X$ is an unknown $n \times p$ matrix, have $a$ solution, is that the matrices

$$
Q=\sum_{i=1}^{r} A_{i}{ }^{T}\left\langle B_{i}\right\rangle \text { and }\left(\begin{array}{c}
C^{R} \\
Q
\end{array}\right), \quad C \neq 0,
$$

have the same rank; and in case $C=0$, a necessary and sufficient condition that the equation have an solution $X \neq 0$ is that $Q$ be of rank less than $n p$.

† Bôcher, loc. cit. 
Equation (12) may be replaced by the equation

$$
\left[\sum_{i=1}^{r}\left\langle A_{i}\right\rangle B_{i}{ }^{T}\right] X^{C}=C^{C},
$$

or the transpose of (12), or that of the present equation. Hence any one of the four matrices,

$$
\sum_{i=1}^{r} A_{i}{ }^{T}\left\langle B_{i}\right\rangle, \quad \sum_{i=1}^{r}\left\langle A_{i}\right\rangle B_{i}{ }^{T}, \quad \sum_{i=1}^{r} A_{i}\left\langle B_{i}{ }^{T}\right\rangle, \quad \sum_{i=1}^{r}\left\langle A_{i}{ }^{T}\right\rangle B_{i},
$$

may be regarded as that of the coefficients of the elements of $X$, that is, as Sylvester's nivellateur. $\dagger$ It should be noted that a different arrangement of the elements of $X$ from that given by either $X^{R}$ or $X^{c}$ or their transposes will result in another matrix which can be obtained from one of the above by an appropriate interchange of rows or of columns. $\$$

Corollary. If $A_{1} B_{1}+A_{2} B_{2}+\cdots+A_{r} B_{r}=0$, where $A_{i}$ ' $(i=1,2, \cdots, r)$, are $m \times n$ matrices and $B_{i}$ are $n \times p$ matrices' then each of the matrices

$$
\sum_{i=1}^{r} A_{i}{ }^{T}\left\langle B_{i}\right\rangle, \quad \sum_{i=1}^{r}\left\langle A_{i}\right\rangle B_{i}
$$

is at most of rank $n^{2}-1$.

This corollary is a direct result of Theorem 4, for here $X=I_{n}$ satisfies the equation (13). It states, perhaps, the most that may be said of such an equation if the $B$ 's are not commutative. Phillips§ gives a more restrictive result in case the $B$ 's are commutative.

The UnIVERSITy OF Wisconsin,

Extension Center, Milwaukee, Wisconsin

† Sylvester, Comptes Rendus, vol. 49 (1884), pp. 409-412, 432-436; Mathematical Papers, vol. 4, pp. 199-205.

$\ddagger$ MacDuffee, loc. cit., was perhaps the first to recognize Sylvester's nivellateur as the sum of direct product matrices, but the matrix 46.2 as he gave it cannot answer the purpose and should be replaced by one of the four matrices in (14). Likewise in his theorem 46.31, the Sylvester nivellateur should be $A\langle I\rangle+I\left\langle B^{T}\right\rangle$ or $\left\langle A^{T}\right\rangle I+\langle I\rangle B$ or the transpose of either of these.

$\S$ Phillips, Functions of matrices, American Journal of Mathematics, vol. 41 (1919), pp. 266-278. 